MATHEMATICS OF COMPUTATION

Volume 77, Number 264, October 2008, Pages 2455-2459

S 0025-5718(08)02134-0

Article electronically published on May 20, 2008

\title{
PRIME FACTORS OF CONSECUTIVE INTEGERS
}

\author{
MARK BAUER AND MICHAEL A. BENNETT
}

\begin{abstract}
This note contains a new algorithm for computing a function $f(k)$ introduced by Erdős to measure the minimal gap size in the sequence of integers at least one of whose prime factors exceeds $k$. This algorithm enables us to show that $f(k)$ is not monotone, verifying a conjecture of Ecklund and Eggleton.
\end{abstract}

\section{INTRODUCTION}

A classic theorem of Sylvester [15] (see also [13]) states that if $n$ and $k$ are positive integers, with $n>k$, then the product of $k$ consecutive integers

$$
\Pi_{n, k}=n(n+1) \cdots(n+k-1)
$$

is necessarily divisible by a prime $p>k$ (i.e. $P\left(\Pi_{n, k}\right)>k$, where $P(m)$ denotes the greatest prime divisor of a positive integer $m$ ). If we therefore, following Erdös [6], define $f(k)$ to be the least integer with the property that

$$
P\left(\Pi_{n, f(k)}\right)>k
$$

whenever $n>k$, it follows that $f(k) \leq k$. Asymptotically, one may in fact show that

$$
\frac{\log k(\log \log k)(\log \log \log \log k)}{(\log \log \log k)^{2}} \ll f(k) \ll \frac{k \log \log \log k}{\log k(\log \log k)},
$$

via work of Rankin 12] and Shorey [14, respectively. Standard heuristics for the size of gaps between consecutive primes lead one to expect that, in fact, the actual order of magnitude of $f(k)$ is $(\log k)^{2}$.

The explicit computation of $f(k)$ for $k \leq 10$ was carried out by Utz [16] (resolving a conjecture of Erdős [6]) and subsequently extended to $k \leq 42$ by Lehmer [11, to $k \leq 46$ by Ecklund and Eggleton [2, and to $k \leq 73$ by Ecklund, Eggleton and Selfridge [3], 4]. We tabulate these results as follows:

\begin{tabular}{|cc|cc|cc|}
\hline$k$ & $f(k)$ & $k$ & $f(k)$ & $k$ & $f(k)$ \\
\hline 1 & 1 & $5-12$ & 4 & $47-58$ & 8 \\
2 & 2 & $13-40$ & 6 & $59-60$ & 9 \\
$3-4$ & 3 & $41-46$ & 7 & $61-73$ & 14 \\
\hline
\end{tabular}

In [2, Ecklund and Eggleton posed the question of whether or not $f(k)$ is monotone and conjectured that it is not; in fact, they expressed a strong belief that $f(113)=14$ and $f(114)=13$ (and that $f(127)=12$ ). In this short note, we

Received by the editor March 14, 2007.

2000 Mathematics Subject Classification. Primary 11N25, Secondary 11D09.

The authors were supported in part by grants from NSERC.

(C)2008 American Mathematical Society Reverts to public domain 28 years from publication 2455 
will present a new algorithm for computing $f(k)$ which will enable us to confirm Ecklund and Eggleton's suspicions. In particular, we prove the following:

Theorem 1.1. If $74 \leq k \leq 178$, then the values of $f(k)$ are as follows:

\begin{tabular}{|cc|cc|}
\hline$k$ & $f(k)$ & $k$ & $f(k)$ \\
\hline $74-113$ & 14 & $115-150$ & 12 \\
114 & 13 & $151-178$ & 14 \\
\hline
\end{tabular}

The fact that $f(114)<f(113)$ contradicts an assertion of Utz $[16]$ to the effect that "if $k$ is composite, the value of $f(k)$ is that of $f(p)$, where $p$ is the largest prime smaller than $k$ ". That this statement might potentially be false was already noted by Ecklund and Eggleton [2].

\section{The ALGORITHM}

For fixed $m$ and $t$, we will in fact consider the problem of classifying those positive integers $n$ for which

$$
P\left(\Pi_{n, m}\right) \leq p_{t}
$$

where $p_{t}$ is the $t$-th prime.

Instead of proceeding as in Lehmer [11, where inequality (2.1) is first treated in case $m=2$ and then extended to larger values of $m$, we will argue as follows. For any given $m \geq 3$, we will split $4[m / 4]$ (or $m-1$, if $m \equiv-1(\bmod 4))$ of the indices $0, \ldots, m-1$ into disjoint pairs of the form $(i, i+2)$, where $i \equiv 0,1(\bmod 4)$. There are

$$
\left[\frac{m}{4}\right]+\left[\frac{m+1}{4}\right]
$$

such pairs. Choose $t_{0}$ such that

$$
\pi(m-1) \leq t_{0} \leq t
$$

If $m \geq 3$, we can thus find $i \in[0, m-3]$ such that the product $(n+i)(n+i+2)$ is divisible by at most

$$
N=\left[\frac{t-t_{0}}{\left[\frac{m}{4}\right]+\left[\frac{m+1}{4}\right]}\right]
$$

of the primes in

$$
\left\{p_{t_{0}+1}, \ldots, p_{t}\right\} \text {. }
$$

Writing $D>1$ for the largest square-free integer dividing $(n+i)(n+i+2)$ and setting

$$
D Y^{2}=(n+i)(n+i+2) \text { and } X=n+i+1,
$$

we find that $X^{2}-D Y^{2}=1$, whereby, if $\left(T_{1}, U_{1}\right)$ are the smallest positive integers for which $x^{2}-D y^{2}=1$, there necessarily exists a positive integer $d$ for which $(X, Y)=\left(T_{d}, U_{d}\right)$, where

$$
T_{d}+\sqrt{D} U_{d}=\left(T_{1}+\sqrt{D} U_{1}\right)^{d} .
$$

Classic results on primitive divisors (see e.g. Carmichael 1]; in this setting, this probably dates back rather earlier to Lucas) ensure that, at least for $d>12$, there is a prime dividing $U_{d}$ that does not divide $D U_{j}$ for $j<d$. Since work of Lehmer 
9] guarantees that if an odd prime $p$ divides some $U_{d}$, then it necessarily divides $U_{j}$ where

$$
j \mid \frac{p-\left(\frac{D}{p}\right)}{2},
$$

we conclude, from the assumption $P\left(U_{d}\right) \leq p_{t}$, that

$$
d \leq \max \left\{\frac{p_{t}+1}{2}, 12\right\} .
$$

Our algorithm thus proceeds as follows. For each of the

$$
M=-1+2^{t_{0}} \sum_{j=0}^{N}\left(\begin{array}{c}
t-t_{0} \\
j
\end{array}\right)
$$

values of $D$ under consideration (trivially omitting $D=1$ ), we first compute the corresponding fundamental solution $\left(T_{1}, U_{1}\right)$. Since we assume that $P\left(T_{d} U_{d} D\right) \leq p_{t}$ and since $T_{1} U_{1} \mid T_{d} U_{d}$, we need only consider those $D$ for which both $P\left(T_{1} U_{1}\right) \leq p_{t}$ and with $D U_{1}$ divisible by at most $N$ of the primes in (2.3). For each such $D$, we can compute $\left(T_{d}, U_{d}\right)$ for all $d$ satisfying (2.5), via recursion say, and check whether $P\left(T_{d} U_{d}\right) \leq p_{t}$ and whether $D U_{d}$ is divisible by at most $N$ primes in (2.3). Actually, we can usually get away with restricting our attention to prime values of $d$ (since $T_{p} U_{p}$ divides $T_{d} U_{d}$ whenever $p \mid d$ ), and moreover employ (2.4) to further reduce our computations.

Essentially, what this algorithm accomplishes is to find all positive integers $r$ such that $P\left(r^{3}-r\right) \leq p_{t}$, under the additional constraint that $r^{2}-1$ is divisible by at most $N$ primes in (2.3). Having carried this out, in order to find all solutions to (2.1), it is left to see whether $P(r \pm j) \leq p_{t}$ holds for at least $m$ consecutive integers of the form $r \pm j$ satisfying $j \leq m-2$. Each $r$ satisfying these criteria yields an integer $n$ such that $P\left(\Pi_{n, m}\right) \leq p_{t}$, and possibly several such $n$ with $n \in[r-(m-2), r+(m-2)]$.

The calculation of $f(k)$ proceeds in two stages from here. Using the list of integers $r$ that are output by the algorithm, we can find all integers $n$ satisfying $P\left(\Pi_{n, m}\right) \leq k$. If all such $n$ are at most $k$ in size, then this establishes an upper bound for $f(k)$, namely, $f(k) \leq m$. If we already know (say via naive search) an integer $n>k$ such that $P\left(\Pi_{n, m-1}\right) \leq k$, then $f(k) \geq m$ and we may conclude that $f(k)=m$. Otherwise, we rerun the algorithm to classify those integers with $P\left(\Pi_{n, m-1}\right) \leq k$, and, if necessary, iterate the process, at each stage replacing $m-i$ by $m-i-1$.

Explicitly, we merely examine all $n$ with this property, and find, for each such $n$, the largest integer $m_{n}$ with the property that $P\left(\Pi_{n, m_{n}}\right) \leq k$. Then $f(k)=$ $\max _{n}\left\{m_{n}\right\}+1$.

\section{Computations}

By way of example, to find the values of $n$ for which $P\left(\Pi_{n, 12}\right) \leq 173$, we take $t=40$ and $m=12$. Our goal is to choose $t_{0}$ in such a way that the number of discriminants we need to consider, $M$ as defined in (2.6), is minimized. In general, it is easy to show that the optimal choice for $t_{0}$ comes from taking either $t_{0}=\pi(m-1)$ 
or from choosing $t_{0}$ to be the minimal integer with $t_{0} \geq \pi(m-1)$ satisfying

$$
t_{0} \equiv t+1\left(\bmod \left[\frac{m}{4}\right]+\left[\frac{m+1}{4}\right]\right) .
$$

The case $(t, m)=(40,12)$ leads to $t_{0}=5$, with a corresponding number of discriminants $M=12293376$.

Proceeding as described above, we find that 2692 of these discriminants yield a suitable $T_{1}$ and $U_{1}$ satisfying both $P\left(T_{1} U_{1}\right) \leq 173$ and $D U_{1}$ divisible by at most 5 of the primes between 13 and 173. Corresponding to these, we find that 183 pairs $\left(T_{d}, U_{d}\right)$ (10 of which have $d>1$ ) lead to $n$ with $P\left(\Pi_{n, 12}\right) \leq 173$. The values for $r$ found using the algorithm satisfy $r \leq 175,295 \leq r \leq 303$ and $319 \leq r \leq 327$. Calculating the corresponding $n$ enables us to conclude as follows :

Proposition 3.1. The positive integer $n$ for which

$$
P\left(\Pi_{n, 12}\right) \leq 173
$$

are precisely those with either $n \leq 167$ or $n \in\{294,295,318,319\}$.

From our remarks at the close of the preceding section and the fact that

$$
P\left(\Pi_{294,13}\right)=151 \text { and } P\left(\Pi_{318,13}\right)=163 \text {, }
$$

we conclude that $f(k)=14$ for $151 \leq k \leq 178$. Similarly, from $P\left(\Pi_{200,11}\right)=103$ and $P\left(\Pi_{114,13}\right)=61$, it follows that, for $61 \leq k \leq 113$, we have $f(k)=14$, while $f(114)=13$ and, for $115 \leq k \leq 150, f(k)=12$, as suspected by Ecklund and Eggleton. This completes the proof of Theorem 1.1.

\section{Concluding Remarks}

The computations leading to Proposition 3.1 were split and run in parallel (the algorithm is clearly well suited to parallelization) on a Mac PowerPC dual core 2.5 $\mathrm{GHz}$ processor, taking a total of just over 58 hours of processing time. Computationally, as one might expect, the costly step is the computation of fundamental units in real quadratic fields; the complexity of this part of the algorithm is well understood (see e.g [17]). The number of fields under consideration, $M$, is typically, for a good choice of $t_{0}$, substantially smaller than $2^{t}-1$, the analogous quantity in Lehmer's approach (at least provided $k>3$ ). Indeed, if we suppose that $t$ is a fixed constant multiple of $m$, say $t=\kappa m$ with $\kappa>1$, and take $t_{0}=\pi(m-1)$, then we have

$$
M=2^{(1+o(1)) \frac{m}{\log m}},
$$

which compares rather favorably with $2^{\kappa m}$.

\section{REFERENCES}

[1] R. D. Carmichael. On the numerical factors of arithmetic forms $\alpha^{n} \pm \beta^{n}$. Ann. Math. 15 (1913), 30-70. MR.1502458

[2] E. F. Ecklund, Jr. and R. B. Eggleton. Prime factors of consecutive integers. Amer. Math. Monthly 79 (1972), 1082-1089. MR0318050 (47:6599)

[3] E. F. Ecklund, Jr., R. B. Eggleton and J. L. Selfridge. Factors of consecutive integers. Proc. Man. Conference Numerical Maths., Winnipeg (1971), 155-157. MR0329183 (48:7525)

[4] E. F. Ecklund, Jr., R. B. Eggleton and J. L. Selfridge. Consecutive integers all of whose prime factors belong to a given set. Proc. Man. Conference Numerical Maths., Winnipeg (1971), 161-162. MR0463097 (57:3059)

[5] R. B. Eggleton and J. L. Selfridge. Consecutive integers with no large prime factors. J. Austral. Math. Soc. Ser. A 22 (1976), no. 1, 1-11. MR0439778(55:12662) 
[6] P. Erdős. On consecutive integers. Nieuw Arch. Wisk. 3 (1955), 124-128. MR0073628 $(17: 461 f)$

[7] P. Erdős and R. L. Graham. Old and New Problems and Results in Combinatorial Number Theory. Monograph Enseign. Math. 28, Geneva, 1980. MR.592420 (82j:10001)

[8] R. K. Guy. Unsolved Problems in Number Theory. 3rd ed. Springer, 2004. MR2076335 (2005h:11003)

[9] D. H. Lehmer. An extended theory of Lucas' functions. Ann. Math. 31 (1930), 419-448. MR.1502953

[10] D. H. Lehmer. On a problem of Störmer. Illinois J. Math. 8 (1964), 57-79. MR0158849 $(28: 2072)$

[11] D. H. Lehmer. The prime factors of consecutive integers. Amer. Math. Monthly 72 (1965), 19-20. MR.0171739 (30:1966)

[12] R. Rankin. The difference between consecutive prime numbers. J. London Math. Soc. 13 (1938), 242-247.

[13] J. Schur. Einege Sätze über Primzahlen mit Anwendung auf Irreduzibilitätsfragen. S.-B. Deutsch. Akad. Wiss Berlin Kl. Math. Phys. Tech. 23 (1929), 1-24.

[14] T. N. Shorey. On gaps between numbers with a large prime factor II. Acta Arith. 25 (1974), 365-373. MR0344201 (49:8941)

[15] J. J. Sylvester. On arithmetical series. Messenger Math. 21 (1892), 1-19, 87-120.

[16] W. R. Utz. A conjecture of Erdős concerning consecutive integers. Amer. Math. Monthly 68 (1961), 896-897. MR0130849 (24:A703)

[17] H. C. Williams. Solving the Pell equation. Number theory for the millennium, III (Urbana, IL, 2000), 397-435, A K Peters, Natick, MA, 2002. MR1956288(2003m:11051)

Department of Mathematics, University of Calgary, Calgary AB

E-mail address: mbauer@math.ucalgary.ca

Department of Mathematics, University of British Columbia, Vancouver BC

E-mail address: bennett@math.ubc.ca 